\title{
Little strong evidence to base treatment of symptomatic lichen planus
}

Chan ES-Y, Thornhill M, Zakrzewska J. Interventions for treating oral lichen planus. The Cochrane Library 1999; 2:1

Objectives To assess the effectiveness and safety of any form of palliative therapy against placebo for the treatment of symptomatic oral lichen planus.

Data sources Search strategy: Medline 1966-98, EMBASE 1980-98, Cochrane Library, handsearching of conference proceedings and specific journals, researchers in the field, drug manufacturers. Selection criteria were any placebo-controlled trial of palliative therapy for symptomatic oral lichen planus, using a randomised or quasirandomised design that measured changes in symptoms and/or clinical signs.

Data extraction and synthesis Change in symptoms (pain, discomfort) and clinical signs (visual impression, lesion measurements) at the end of therapy. Odds ratio of improvement versus no improvement for each trial outcome and pooling where appropriate.

Results Nine therapies identified grouped into four separate classes (cyclosporines, retinoids, steroids and phototherapy) for comparison.
No therapy was replicated exactly. Large odds ratios with very wide confidence intervals indicating a statistically significant treatment benefit were seen in all trials. However, this has to be tempered by considerations of the small study sizes, lack of replication, difficulty in measuring outcome changes and the very high likelihood of publication bias. Only systemic agents were associated with treatment toxicities, all other side-effects were mild and mainly limited to local mucosal reactions.

Conclusions The review provides only weak evidence for the superiority of the assessed interventions over placebo for palliation of symptomatic OLP. The results highlight the need for larger placebocontrolled RCTs with more carefully selected and standardised outcome measures before between-treatment comparisons can be properly interpreted.

Address for reprints: The Cochrane Library, UK Cochrane Centre, Summertown Pavillion, Middle Way, Oxford OX2 7LG, UK.

\section{Commentary}

This review from the Cochrane database surveys randomised placebo-controlled trials of palliative treatment for symptomatic oral lichen planus. Nine studies were identified that withstood the rigorous selection criteria. Four treatment modalities were tested: topical steroids (probably the most-used modality in clinical practice), topical cyclosporine rinses (an interesting but costly approach with theoretical appeal), topical and systemic retinoids (drugs used mostly by dermatologists at present), and PUVA (phototherapy that requires special equipment and that might be unacceptably risky for routine use). The authors felt that the lack of evidence of efficacy of any active treatments meant that it was difficult to compare treatments and therefore they looked at the placebocontrolled trials first and each treatment is compared only with placebo.
Each reported trial indicated a statistically significant clinical improvement from the tested treatment. Despite positive results, the authors rightly propose a very conservative interpretation in their discussion. None of the reported trials enrolled large numbers of subjects, and the confidence intervals were very broad, indicating wide variability in outcome and/or a lack of measurement precision. This is expected, given the inherent problems in lichen planus studies; no practice sees large numbers of symptomatic patients and there is no consensus as to what constitutes a successful treatment outcome or how results ought to be measured. More important perhaps is the authors' caveat that reporting bias could well be at work. Negative results might be under-reported, and this could alter the clinical applicability of these results. The most valuable points in this analysis are the conclu- sions and implications for future research: We have relatively little strong evidence at the present time upon which to base treatment decisions for our patients suffering with symptomatic lichen planus. The research community needs to standardise protocols for diagnosis, assessment of symptoms and signs, and outcomes measurement for oral lichen planus. This might best be accomplished by a working group and publishing workshop proceedings. Multicentre randomised controlled trials could focus on the identification of effective therapy. This important review identifies a clear unmet need in the area of clinical research on oral disease.

\section{George T Gallagher \\ Dept of Oral and Maxillofacial \\ Pathology, Harvard School of Dental \\ Medicine, Boston, USA}

